\title{
High-resolution reconstruction and deconvolution of array detector images
}

\author{
Sylvain Prigent ${ }^{1}$, Stéphanie Dutertre ${ }^{2}$, Aurélien Bidaud-Meynard ${ }^{3}$, \\ Giulia Bertolin ${ }^{3}$, Grégoire Michaux ${ }^{3}$, and Charles Kervrann ${ }^{1}$ \\ ${ }^{1}$ Inria Centre Rennes-Bretagne Atlantique, France \\ ${ }^{2}$ Univ Rennes, UMS Biosit, MRic, F-35000 Rennes, France \\ ${ }^{3}$ Univ Rennes, CNRS, IGDR (Genetics and Development Institute \\ of Rennes), UMR 6290, F-35000 Rennes, France \\ ${ }^{*}$ Corresponding author: sylvain.prigent@inria.fr
}

August 2, 2021

\begin{abstract}
Array detector allows a resolution gain for confocal microscopy by combining several images acquired by 32 sub-detectors. A single high resolution image is generally obtained by spatially re-assigning the signal from the 32 sub-detectors and applying a deconvolution procedure. Alternative methods have been proposed to produce high resolution images by linearly combining the signals of sub-detectors. We propose here a novel method that deconvolves directly the stack constituted of 32 spatially re-assigned detectors signals. We demonstrate on both calibration slides and real data that the proposed method allows to produce more homogeneous, well-contrasted, high-resolution images than those obtained with previous methods. All the tested methods have been implemented in an open source software which can be be downloaded for free.
\end{abstract}

The principle of array detector confocal microscopy is to replace the point detector by a set of 32 detectors. As illustrated in Fig. 1, the 32 sub-detectors, labeled with numbers 1-32, are generally organized around a central sub-detector [1. Each individual sub-detector has a diameter of $0.2 \mathrm{AU}$ (Airy Unit). The inner hexagonal patch gathering the 7 central detectors has a diameter of 0.6 AU. The inner ring gathering the sub-detectors 8-19 has a diameter of 1 AU. The whole array of 32 sub-detectors has a diameter of $1.25 \mathrm{AU}$. Unlike conventional confocal microscopy, the spatial resolution of images is no longer limited by the size of the pinhole.The resulting images have a higher signal-to-noise ratio (SNR) when compared to images acquired in confocal microscopy. Nevertheless the reconstruction of a single high-resolution image from 32 sub-detectors is not straightforward. Two categories of approaches are generally considered to 


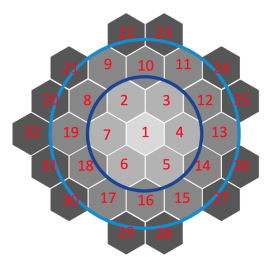

Figure 1: Scheme of the array detector. The 32 sub-detectors are hexagonal and arranged around the central sub-detector 1 . The numbers are the sub-detectors indexes. The dark blue circle delineates the group of 7 central detectors and the light blue circle represents the border of the group of 19 central detectors.

address the underlying computational issue. The first category of approaches amounts to appropriately fusing the 32 images. Techniques based on linear combination of sub-detectors, spatial re-assignment, or virtual emission difference have been proposed, and are discussed in the letter. These techniques allows either a gain of contrast or a gain of resolution, but not both. The second category of methods relies on deconvolution of the measured signals. The related algorithms are able to improve the contrast and the resolution of images, but also may create undesirable artifacts in the reconstructed image.

In this letter, we first present the techniques based on linear combination of sub-detectors, as well as a recent method used to automatically select the optimal parameters controlling the underlying weighted averages. In the second part of the letter, we describe a new deconvolution technique to estimate a single high-resolution image directly from the array of 32 images. We quantitatively demonstrate the performance of our computational method on calibration slides and real data and we compare the results to those obtained with all aforementioned techniques.

Formally, we denote $N_{c_{1}}=7$ and $N_{c_{2}}=19$ the number of central subdetectors of order 1 and order 2, respectively, and $N_{T}=32$ is the total of sub-detectors. The simplest reconstruction method to create a pseudo-confocal (PC) image amounts to summing the intensity values of $n=\{7,19,32\}$ subdetectors at each spatial position in the image domain $\Omega$ :

$$
\hat{u}_{P C}(x)=\sum_{i=1}^{n} I_{i}(x)
$$

where $I_{i}(x)$ is the intensity value measured at spatial position $x \in \Omega$ and $i$ is the index of a given sub-detector (see Fig. 1). We obtain a reconstructed image equivalent to a confocal image with a pinhole at $0.6 \mathrm{AU}, 1 \mathrm{AU}$, and $1.25 \mathrm{AU}$, by setting $n=7,19$ and 32, respectively. Whatever $n$ is, the reconstructed image has a higher contrast than a conventional confocal image.

In recent years, more sophisticated combinations of sub-detectors have been investigated to produce high-resolution images. First, Image Scanning Microscopy (ISM) consists in reassigning the signal from each sub-detector to the 
central detector and summing the $N_{T}$ registered intensity values as follows:

$$
\hat{u}_{I S M}(x)=\sum_{i=1}^{N_{T}} \tilde{I}_{i}(x)
$$

where $\tilde{I}_{i}(x)$ denotes the intensity value at pixel $x$ attached to sub-detector $i$ which is spatially co-registered to the central detector [2, 3. Virtual Fluorescence Emission Difference (FED) has been proposed in [4, 5] to create a FED image from an array detector. FED microscopy scans twice the sample with two different illumination shapes: a point shape and a donut shape. The FED image is obtained by subtracting the outer-ring of sub-detectors to the inner detectors:

$$
\hat{u}_{I F E D}(x)=\sum_{i=1}^{n} I_{i}(x)-\epsilon \sum_{i=n+1}^{N_{T}} I_{i}(x),
$$

where $\epsilon>0$ is a subtracting factor and $n=7$ or 19 , depending on the desired compromise between contrast and resolution. In [6], the authors experimentally showed that combining ISM and IFED as follows:

$$
\hat{u}_{I S F E D}(x)=\sum_{i=1}^{N_{T}} \tilde{I}_{i}(x)-\epsilon \sum_{i=1}^{N_{T}} I_{i}(x), \epsilon>0,
$$

significantly improves the resolution of the reconstructed image (about $12 \%$ in [6]).

In all cases, the aforementioned IFED and ISFED methods need the operator to manually tune the $\epsilon$ parameter. In [5], the authors found that setting $\epsilon$ to 0.3 enables to achieve good results, for low SNR values. Nevertheless, for high SNR values, the reconstruction results may be improved if the value $\epsilon$ is increased (e.g., $\epsilon=0.9$ ). We thus proposed in 7 a data-driven method to automatically set $\epsilon$, and which is summarized below. First, we formally generalize IFED and ISFED by using the following formulation:

$$
\hat{u}(x)=K_{\rho} * \sum_{i=1}^{m_{1}} I_{i}(x)-\epsilon K_{\rho} * \sum_{i=m_{2}}^{N_{T}} I_{i}(x) \triangleq a_{I}(x)-\epsilon b_{I}(x)
$$

where $\rho$ is the bandwidth of a spatial convolution filter $K$ used for noise reduction, $*$ denotes the convolution operator, $a_{I}(x)$ is the sum of the sub-detectors with indexes ranging from 1 to $m_{1}, b_{I}(x)$ is the sum of the sub-detectors with indexes ranging from $m_{2}$ to $N_{T}$, and $\epsilon>0$ as before. It follows that $\hat{u}=u_{I F E D}$ if we set $m_{1}=n$ and $m_{2}=n+1$ in (5), with $n=7$ or 19 . Similarly $\hat{u}=u_{\text {ISFED }}$ if $m_{1}=N_{T}$ and $m_{2}=1$ in (5). The bandwidth $\rho$ is chosen very small to slightly reduce noise while preserving high frequencies. The optimal value of $\epsilon$ is obtained by minimizing the local Stein's unbiased risk $(S U R E)$ [8, 9] while considering the central detector $I_{1}$ as the observation data:

$$
S U R E(x)=\frac{1}{|\Omega(x)|} \sum_{y \in \Omega(x)}\left(I_{1}(y)-\hat{u}(y)\right)^{2}-\sigma^{2}+\frac{2 \sigma^{2}}{|\Omega(x)|} \operatorname{div}_{I_{1}}(\hat{u}),
$$

where $|\Omega(x)|$ is the cardinal of the local neighborhood $\Omega(x)$ centered at point $x \in \Omega, \sigma^{2}$ is the variance of the assumed Gaussian noise, and $\operatorname{div}(\cdot)$ is the 
divergence operator. Solving $\frac{\partial S U R E(x)}{\partial \epsilon}=0$ gives the following closed-form solution for $\epsilon$ :

$$
\epsilon=\frac{\sum_{x \in \Omega}\left[\left(N_{T}-m_{2}\right) \sigma^{2}-|\Omega(x)|^{-1} \sum_{y \in \Omega(x)}\left(I_{1}(y)-a_{I}(y)\right) b_{I}(y)\right]}{\sum_{x \in \Omega}|\Omega(x)|^{-1} \sum_{y \in \Omega(x)} b_{I}^{2}(y)} .
$$

In our experiments, the IFED and ISFED reconstructed images are obtained by automatically setting $\epsilon$ from the solution (7).

The ISM, IFED, and ISFED techniques improve resolution by applying simple linear operations on the sub-detectors signals. Nevertheless, ISM provides a small resolution gain, while IFED and ISFED produce reconstructed images with low SNRs. To overcome these shortcomings, another approach consists in applying a deconvolution algorithm to the ISM image. In what follows, we will denote ISM-RL and ISM-W the image deconvolved with the conventional LucyRichardson (RL) and Wiener (W) algorithms, respectively. Deconvolving the ISM image is however sub-optimal since it is applied to the average of signals from the 32 sub-detectors. Some information is irremissibly lost with this strategy. To remedy this, we describe below a novel array detector deconvolution method directly applied to the full array data.

As the fluorescence images are generally smooth and sparse, and are corrupted by mixed Gaussian-Poisson noise [10, 11, we consider the following deconvolution problem (see deails in Supplementary Material):

$$
\min _{u} R(u) \quad \text { subject to } \quad\|f-(h * u)\|_{2}^{2}=\alpha\|f\|_{1}+|\Omega| \sigma^{2},
$$

where $|\Omega|$ is the number of pixels in $\Omega, f$ represents the blurred and noisy image, $h$ denotes the point spread function (PSF), $u$ is the restored image, $\alpha>0$ is the quantization factor of the photodetector, $\sigma^{2}>0$ encompass the additional approximately Gaussian noises, and $R(u)$ is a regularization term that promotes smoothness and sparsity of the solution. Solving $(19)$ is then equivalent to minimizing the following energy if $f=\hat{u}_{I S M}$ (see Supplementary Material):

$$
\hat{u}=\underset{u}{\operatorname{argmin}} \frac{1}{2}\left\|\hat{u}_{I S M}-(h * u)\right\|_{2}^{2}+\lambda \underbrace{\sqrt{\rho^{2}\|\mathcal{H} u\|_{F}^{2}+(1-\rho)^{2} u^{2}}}_{R(u)},
$$

where $\mathcal{H}$ is the Hessian operator and $\|\cdot\|_{F}^{2}$ denoters the Frobenius norm. The Hessian operator (2nd order derivatives) is recommended here to avoid sharp discontinuities in the reconstructed images [12, 13, 14] and while the second term $u^{2}$ in $R(u)$ is a sparsity term that promotes a flat and large background. The parameters $\lambda$ and $\rho \in[0,1]$ are weighting parameters used to control the amount of smoothness and sparsity in the reconstructed image. The restored image is found by minimizing this energy with a fast primal-dual optimization algorithm (see details in [15. Instead of minimizing (9) to produce the so-called ISMSV (Sparse-Variation) image, we propose below to translate the deconvolution problem into a super-resolution problem and to reconstruct a higher-resolution image from the set images $\left\{I_{i}\right\}_{i=1}^{N_{T}}$ provided by the $N_{T}$ sub-detectors. The novel 
energy to be minimized is then defined as the sum of the previous regularization term and a data fitting term relying on the $N_{T}$ sub-detectors:

$$
\hat{u}=\underset{u}{\operatorname{argmin}} \frac{1}{2} \sum_{i=1}^{N_{T}} d_{i}\left\|I_{i}-(h * u)\right\|_{2}^{2}+\lambda \sqrt{\rho^{2}\|\mathcal{H} u\|_{F}^{2}+(1-\rho)^{2} u^{2}},
$$

where $d_{i}$ is the Euclidean distance of the sub-detector $i$ to the central detector. The periherical images 20-32 contain high spatial frequencies and significantly contribute here to the reconstruction. Unlike ISM-SV, the resulting algorithm (denoted SV) allows one to jointly combine several sub-detectors and perform deconvolution.

In our experiments, we compared the performance of PC, ISM, IFED, ISFED, ISM-RL, ISM-W, ISM-SV and SV algorithms on several stacks of $N_{T}=32$ sub-detectors. First, we applied the eight reconstructions methods to a stack depicting a ring shape with homogeneous intensity, acquired with the Argolight calibration slide 16. In Fig. 2, we can observe that PC and ISM produce images with a high SNR but a low resolution. IFED and ISFED methods produced images with a low SNR due to the sub-detectors subtraction, but the obtained resolution is higher than those observed in PC and ISM images. The three deconvolution methods ISM-RL, ISM-Wiener and ISM-SV provide higher resolution images. Nevertheless, they are sensitive to small intensity variations in the images, and the reconstructed ring is not spatially homogeneous (see second row in Fig. 22. Finally, the ring reconstructed by the SV method is visually thinner and more homogeneous when compared to the other deconvolution methods applied to the ISM image. To provide quantitative evaluation, we plotted 12 profiles across the ring equally separated by an angle of 30 degrees (see Fig. 3). We estimated the average thickness of the ring by using the full-width at half-maximum (FWHM) value. The two peaks are large and noisy on the PC and ISM images and the average thickness are $353 \pm 58 \mathrm{~nm}$ and $308 \pm 14 \mathrm{~nm}$, respectively. The average thickness of the ring are smaller on the IFED $(290 \pm 75 \mathrm{~nm})$ and ISFED $(229 \pm 73 \mathrm{~nm})$ images. The quantitative results are significantly improved when deconvolution is applied to the ISM image. The profiles are smooth and the average thickness are $265 \pm 9 \mathrm{~nm}, 236 \pm 18 \mathrm{~nm}$, and $241 \pm 16 \mathrm{~nm}$ with the ISM-W, ISM-RL and ISM-SV methods, respectively. Finally, the SV reconstruction method provided profiles which are less noisy and thinner. The average thickness is among the smallest $(243 \pm 9 \mathrm{~nm})$, and the variance of the reconstructed image is smaller when compared to the other deconvolution methods tested.

To estimate spatial resolution, we applied the eight reconstruction methods to the Argolight calibration slide [16] depicting vertical lines pattern (see Fig. 4 a). The pattern is constituted of 10 groups of lines where the distance between two consecutive lines decreases from $550 \mathrm{~nm}$ to $100 \mathrm{~nm}$ with a step of $50 \mathrm{~nm}$. Fig. 4 a shows a crop of the line pattern reconstructions. We measured the contrast between the maximum and minimum intensity profiles for each line (see Fig. 4b). As denoted in the calibration slide documentation [16] a measured contrast below $26.5 \%$ means that the objects at the line resolution cannot be 


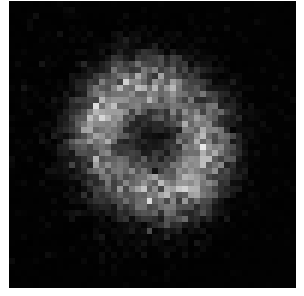

PC

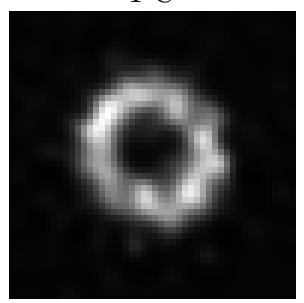

ISM-RL

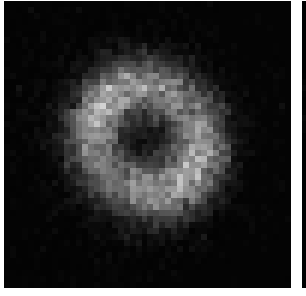

ISM

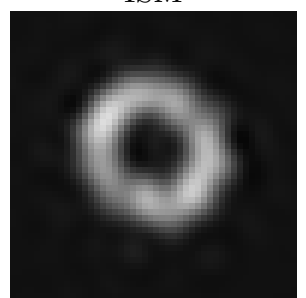

ISM-Wiener

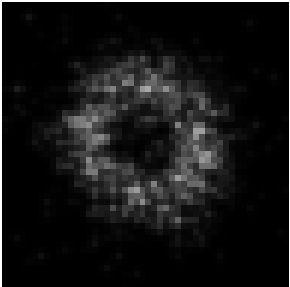

IFED

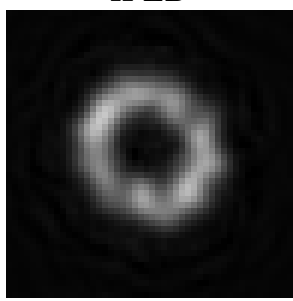

ISM-SV

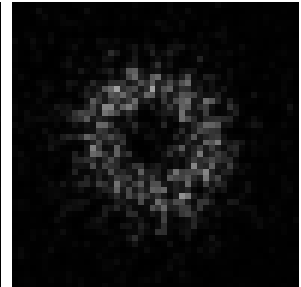

ISFED

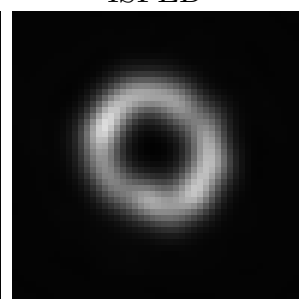

SV

Figure 2: Reconstruction results obtained on a ring shape with homogeneous intensity from the Argolight calibration slide on a ZEISS Airyscan microscope for each studied methods.

distinguished. In our experiments, the PC, ISM and IFED methods cannot separate two lines less than $350 \mathrm{~nm}$ apart. The results are significantly improved by applying the other methods because the resulting resolution is about 250 $\mathrm{nm}$. It is worth noting that the resolution in the ISFED image is similar to the resolution obtained with any deconvolution methods, but the reconstructed ISFED image is noisier.

To evaluate the reconstruction methods on real data, we applied the eight reconstruction methods to an image depicting mitochondria of MCF7 cells expressing mito-GFP (Fig. 5). As before, the ISFED method and the deconvolution methods applied to the ISM image yields the highest resolution images, but the SNR is higher with the deconvolution techniques. The SV method performed best among the deconvolution methods in terms of robustness to noise and resolution. To quantitatively assess resolution, we plotted the intensity profiles along a line displayed in yellow on the reconstructed images shown in Fig. 5. The normalized profiles estimated with each reconstruction method are shown in Fig. 6. The ISFED profile is the thinnest profile but it is much noisier than the other profiles. The SV method achieved the best compromise between SNR and resolution, followed by ISM-SV, ISM-RL, ISM-Wiener, and IFED, ISM and PC.

Finally, we applied the reconstruction methods to an image showing intestinal microvilli from an adult C. elegans worm expressing the ERM-1::mNeonGreen fusion protein; ERM-1 is specifically localized in intestinal microvilli (see Fig. 7). The ISFED method and the deconvolution methods yielded the highest resolution images; the measured size of microvilli is $115 \mathrm{~nm}$ using the FWHM criterion. In Fig. 8), the SV method produced the more contrasted images and 
the deeper intensity profiles measured along the yellow lines displayed on the zoom-in views of each reconstructed images. Furthermore, we performed a second experiment using a $C$. elegans intestinal microvilli sample to evaluate the methods sensitivity to noise. Several images depicting the same sample have been taken with four different pixel dwell times corresponding to different acquisition speeds. Fig. 9 shows the reconstructed PC images and the evolution of SNRs for four different acquisition speeds. Furthermore, we considered the eight reconstruction methods and quantitatively evaluated the contrast (percentage) between microvilli (see plots in Fig. 10. First, the contrast corresponding to PC and ISM imaged, is below 26.5\%, suggesting microvilli cannot be separated whatever the acquisition speed is. Second, microvilli can be isolated with IFED and ISFED methods on images captured with speed \#1 and speeds \#1 and \#2, respectively. Finally, all the deconvolution methods allow to detect the microvilli for any scanning speeds, but the most performant method is SV because it produces the highest contrasted images when the SNR decreases (higher than $40 \%$ for any scanning speeds).

In this letter, we compared the performance of several reconstruction methods able to produce high-resolution confocal images from an array of subdetectors. We proposed a novel array deconvolution method to reduce both noise and blur the reconstructed images. The SV method exploits multiple subdetectors images and performs better than the existing methods on calibrated and well-controlled reference images, as well as on real images. Only one second and two seconds are required for processing a $512 \times 512$ image (core i7 CPU) by using the ISM, IFED, ISFED methods, and the deconvolution methods, respectively. The software that implements all the methods is available as a Fiji plugin at: https://team.inria.fr/serpico/airyscanj/. Note that, the SV method is very general as it can process $3 \mathrm{D}$ images in more conventional fluorescence microscopy.

Funding France-BioImaging infrastructureANR-10-INBS-04.

\section{References}

[1] K. Weisshart, "The basic principle of airyscanning," ZEISS Technology Note, 2014

[2] C. B. Müller and J. Enderlein, "Image scanning microscopy," Phys. Rev. Lett., vol. 104, p. 198101, May 2010.

[3] C. J. R. Sheppard, S. B. Mehta, and R. Heintzmann, "Superresolution by image scanning microscopy using pixel reassignment," Opt. Lett., vol. 38, pp. 2889-2892, Aug 2013

[4] Y. Ma, C. Kuang, Y. Fang, B. Ge, D. Li, and X. Liu, "Virtual fluorescence emission difference microscopy based on photon reassignment," Opt. Lett., vol. 40, pp. 4627-4630, Oct 2015

[5] B. Ge, Y. Wang, Y. Huang, C. Kuang, Y. Fang, P. Xiu, Z. Rong, and X. Liu, "Three-dimensional resolution and contrast-enhanced confocal microscopy with array detection," Opt. Lett., vol. 41, pp. 2013-2016, May 2016.

[6] Y. Li, S. LIU, D. Liu, S. Shiyi, C. KUANG, Z. DING, and X. LIU, "Image scanning fluorescence emission difference microscopy based on a detector array: Isfed based on a detector array," Journal of Microscopy, vol. 266,
022017 .

[7] S. Prigent, S. Dutertre, and C. Kervrann in ICASSP 2020 - 2020 IEEE International Conference on Acoustics, Speech and Signal Processing (ICASSP), pp. 1075-1079, 2020.

[8] F. Luisier, T. Blu, and M. Unser, "SURE-LET for orthonormal wavelet-domain video denoising," IEEE Transactions on Circuits and Systems for Video Technology, vol. 20, pp. 913-919, June 2010.

[9] T. Qiu, A. Wang, N. Yu, and A. Song, "Llsure: Local linear sure-based edge-preserving image filtering," IEEE Transactions on Image Processing, vol. 22, pp. 80-90, Jan 2013. 
bioRxiv preprint doi: https://doi.org/10.1101/2021.08.02.454749; this version posted August 4, 2021. The copyright holder for this preprint (which was not certified by peer review) is the author/funder. All rights reserved. No reuse allowed without permission.

[10] L. M. Mugnier, T. Fusco, and J.-M. Conan, "Mistral: a myopic edge-preserving image restoration method, with application to astronomical adaptive-optics-corrected long-exposure images," J. Opt. Soc. Am. A, vol. 21, pp. 1841-1854, Oct 2004 .

[11] F. Soulez, L. Denis, Y. Tourneur, and E. Thiébaut in 2012 9th IEEE International Symposium on Biomedical Imaging (ISBI), pp. $1735-1738,2012$

[12] S. Lefkimmiatis, A. Bourquard, and M. Unser, "Hessian-based norm regularization for image restoration with biomedical applications," IEEE Trans. Image Processing, vol. 21, pp. 983-995, March 2012.

[13] M. Arigovindan, J. C. Fung, D. Elnatan, V. Mennella, Y.-H. M. Chan, M. Pollard, E. Branlund, J. W. Sedat, and D. A. Agard, "High-resolution restoration of 3D structures from widefield images with extreme low signal-
to-noise-ratio," Proc. Natl. Acad. Sci. USA, vol. 110, no. 43, pp. 17344-17349, 2013 .

[14] H. Ikoma, M. Broxton, T. Kudo, and G. Wetzstein, "A convex 3d deconvolution algorithm for low photon count fluorescence imaging," Scientific Reports, vol. 8, no. 1, p. 111489, 2018

[15] H.-N. Nguyen et al., "Spitfir(e): a supermaneuverable algorithm for 2d-3d+time fluorescence image restoration and background subtraction," in bioRxiv, 2021.

[16] A. Royon and N. Converset, "Quality control of fluorescence imaging systems," Optik \& Photonik, vol. 12, no. 2, pp. $22-25,2017$.

[17] L. I. Rudin, S. Osher, and E. Fatemi, "Nonlinear total variation based noise removal algorithms," Physica D:Nonlinear Phenomena, vol. 60, no. 1/4, pp. 259-268, 1992. 


\section{Supplementary Materials}

\section{Definition of the regularization energy for image deconvolution.}

A fidelity data term is generally derived from the general formation model, for instance dedicated to low photon counts and low-light regimes in fluorescence microscopy (e.g. [14]). We formally demonstrate below that a conventional quadratic fidelity term, which is optimal when the images are corrupted by additive white Gaussian noise, is also appropriate for mixed Poisson-Gaussian noise.

Assume an image $u \in \Omega$ corrupted by a mixed Poisson-Gaussian and denote $f$ the noisy image. The local noise variance can be represented at a given location $\mathbf{x} \in \Omega$ as follows [10, 11]:

$$
V_{\text {noise }}(\mathbf{x}) \triangleq \mathbb{E}\left[(f(\mathbf{x})-(h * U)(\mathbf{x}))^{2}\right]=\alpha u(\mathbf{x})+\sigma^{2},
$$

where $U$ is the random variable associated to the realization $u, \alpha>0$ is the quantization factor of the photodetector and $\sigma^{2}>0$ encompass the additional approximately Gaussian noises. If we assume that the average intensity is preserved, i.e.

$$
\int_{\Omega} u(\mathbf{x}) d \mathbf{x}=\int_{\Omega} f(\mathbf{x}) d \mathbf{x}
$$

it follows that, by pre-multiplying the intensities on both sides of the previous equation by a factor $\alpha$ and adding the constant value $\sigma^{2}$, we have:

$$
\int_{\Omega} \alpha u(\mathbf{x})+\sigma^{2} d \mathbf{x}=\int_{\Omega} \alpha f(\mathbf{x})+\sigma^{2} d \mathbf{x} .
$$

Moreover, by integrating (11) over the image domain $\Omega$, we get

$$
\begin{aligned}
\int_{\Omega} \mathbb{E}\left[(f(\mathbf{x})-(h * U)(\mathbf{x}))^{2}\right] d \mathbf{x} & =\int_{\Omega} \alpha u(\mathbf{x})+\sigma^{2} d \mathbf{x}, \\
\iint_{\Omega}\left(f(\mathbf{x})-\left(h * u^{\prime}\right)(\mathbf{x})\right)^{2} p\left(u^{\prime}(\mathbf{x})\right) d \mathbf{x} d u^{\prime}(\mathbf{x}) & =\int_{\Omega} \alpha u(\mathbf{x})+\sigma^{2} d \mathbf{x},
\end{aligned}
$$

where $p\left(u^{\prime}(\mathbf{x})\right)$ represents the probability distribution of the random variable $U$. Assume the following prior $p\left(u^{\prime}(\mathbf{x})\right)=\mathbf{1}[f(\mathbf{x})=((h * u)(\mathbf{x}))]$ where $\mathbf{1}[\cdot]$ is the indicator function. Hence, we have by using 13 .

$$
\begin{aligned}
\int_{\Omega}(f(\mathbf{x})-(h * u)(\mathbf{x}))^{2} d \mathbf{x} & =\int_{\Omega} \alpha u(\mathbf{x})+\sigma^{2} d \mathbf{x} \\
& =\int_{\Omega} \alpha f(\mathbf{x})+\sigma^{2} d \mathbf{x} \\
\|f-(h * u)\|_{2}^{2} & =\alpha\|f\|_{1}+|\Omega| \sigma^{2}
\end{aligned}
$$

Finally, starting from the seminal paper [17, the restored image is found by solving the following optimization problem (Poisson-Gaussian noise):

$$
\min _{u} R(u) \quad \text { subject to } \quad\|f-(h * u)\|_{2}^{2}=\alpha\|f\|_{1}+|\Omega| \sigma^{2} .
$$

It turns out that 19 is a constrained formulation with an equality constraint which is not convex. The corresponding formulation with inequality constraint is

$$
\min _{u} R(u) \quad \text { subject to } \quad\|f-(h * u)\|_{2}^{2} \leq \alpha\|f\|_{1}+|\Omega| \sigma^{2}
$$


It has been established that, under additional assumptions, that 19 and $(20)$ are equivalent. A Lagrange formulation can be then derived since the the right-hand side of the inequality does not depend on the unknown image $u$ :

$$
\min _{u} R(u)+\lambda^{\prime}\|f-(h * u)\|_{2}^{2}
$$

where the parameter $\lambda^{\prime}>0$ balances the two energy terms and is an unknown function of the Poisson-Gaussian noise variance. The Karush-Tucker conditions guarantees that (22) and 20 are equivalent for a particular choice of $\lambda^{\prime}$. Finally, if we set $\lambda^{\prime}=\lambda^{-1} \neq 0$, we can equivalently reformulate the minimization problem as:

$$
\min _{u}\|f-(h * u)\|_{2}^{2}+\lambda R(u)
$$




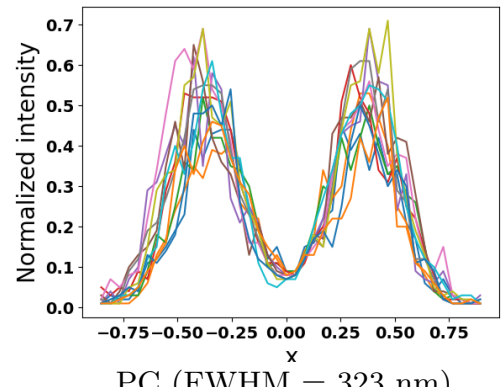

$\mathrm{PC}(\mathrm{FWHM}=323 \mathrm{~nm})$

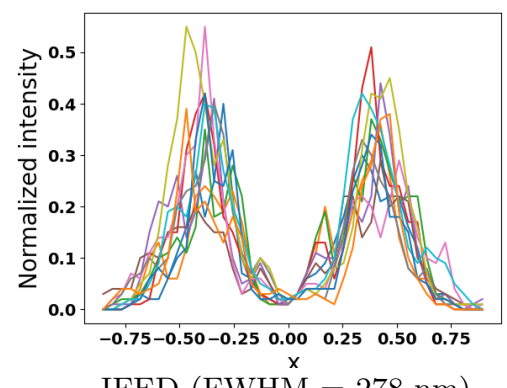

IFED $(\mathrm{FWHM}=278 \mathrm{~nm})$

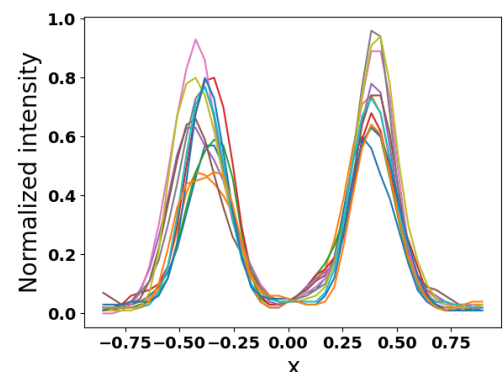

ISM-RL $(\mathrm{FWHM}=257 \mathrm{~nm})$

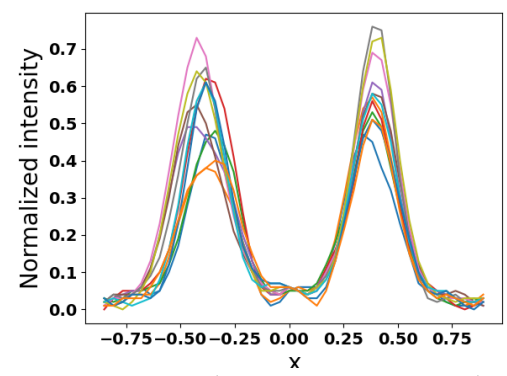

ISM-SV $($ FWHM $=254 \mathrm{~nm})$

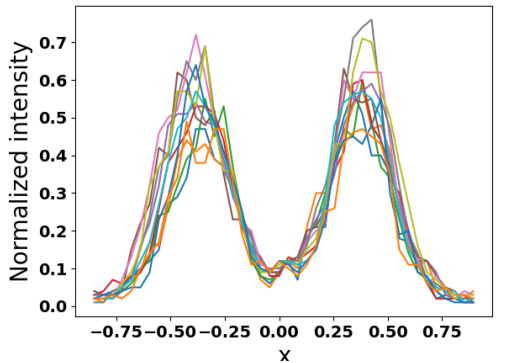

ISM $(\mathrm{FWHM}=313 \mathrm{~nm})$

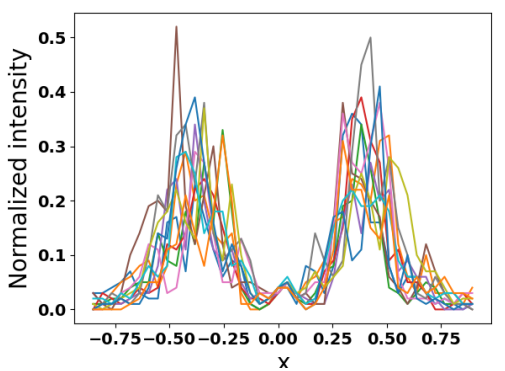

ISFED $(\mathrm{FWHM}=280 \mathrm{~nm})$

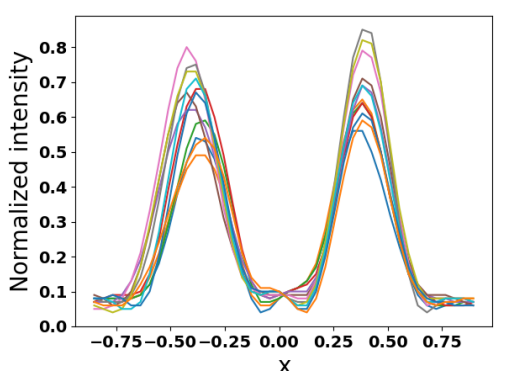

ISM-W $(\mathrm{FWHM}=275 \mathrm{~nm})$

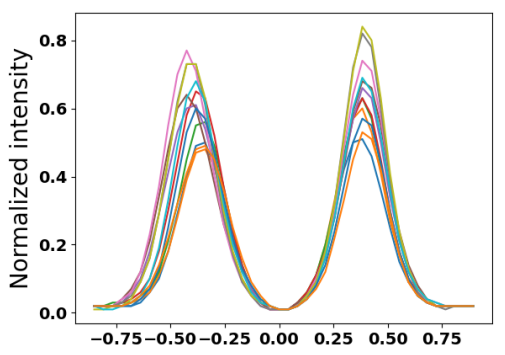

SV $\left(\right.$ FWHM $\left.^{\mathrm{x}}=252 \mathrm{~nm}\right)$

Figure 3: Profile plots across the ring of Fig. 2 for each reconstruction methods. 12 profiles are plot across the ring equally separated by an angle of 30 degrees. The $x$ axis represents the distance (in microns) to the ring center. 

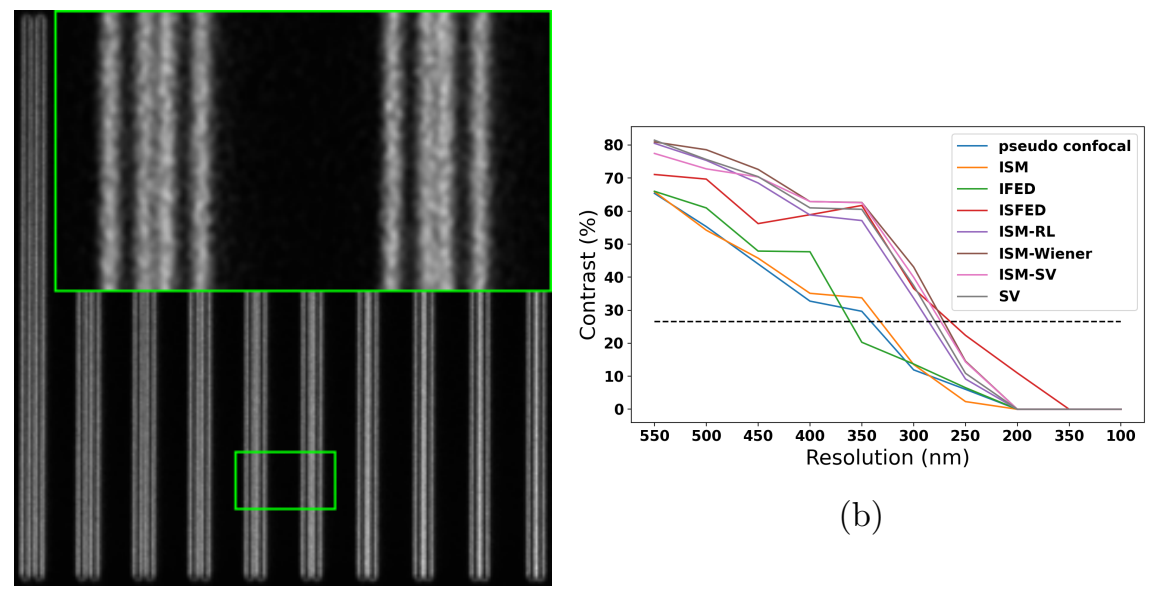

(b)

(a)

Figure 4: Resolution measurement using the vertical line pattern. (a) Pattern image and zoom-in view: from left to right, lines are spaced from $550 \mathrm{~nm}$ to $100 \mathrm{~nm}$ by step of $50 \mathrm{~nm}$. (b) Contrast in percentage of intensity between the maximum and minimum intensities for the 10 vertical lines patterns profiles. The black dashed line shows the resolution measurement threshold. The resolution is measured at the cross of the curve and the black dashed line.

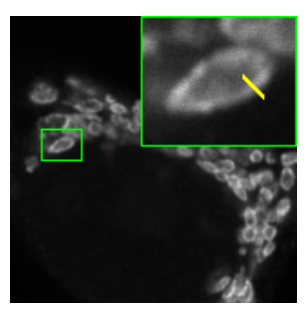

PC

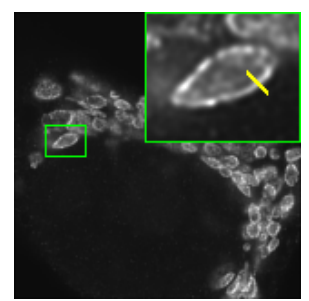

ISM-RL

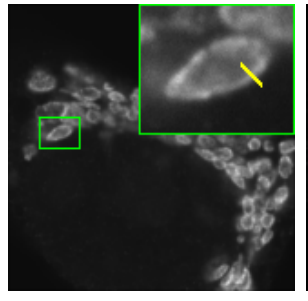

ISM

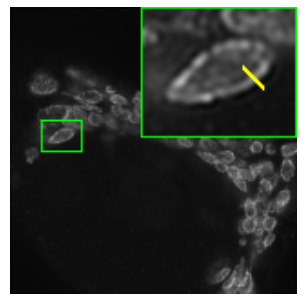

ISM-W

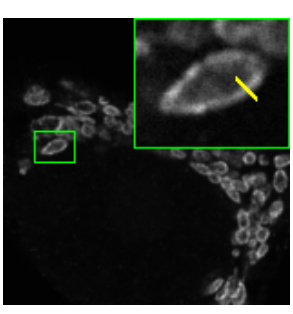

IFED

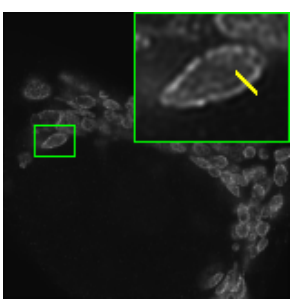

ISM-SV

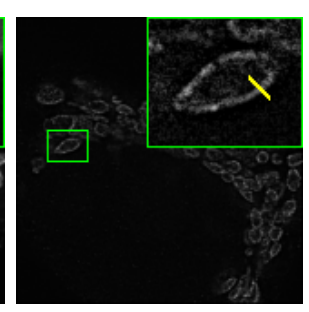

ISFED

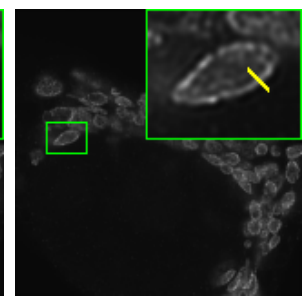

SV

Figure 5: Reconstruction results obtained on MCF7 cells expressing mito-GFP (labelling mitochondria). A line perpendicular across a cell membrane is displayed on zoom-in views of each reconstructed images. 


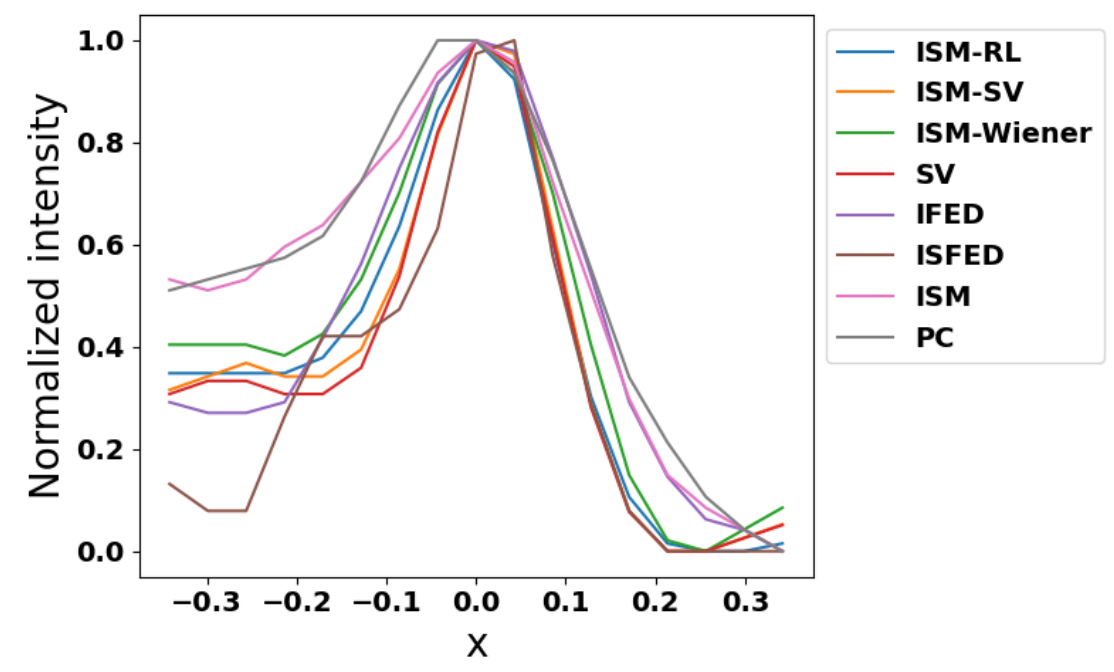

Figure 6: Intensity profiles along the yellow line displayed in Fig. 5 for each reconstruction method. The $x$ axis is graduated as the distance (in microns) to the line center. For each reconstruction method we measure a FWHM of: 272 for PC, 260 for ISM, 207 for IFED, 117 for ISFED, 160 for ISM-RL, 188 for ISM-W, 150 for ISM-SV and 147 for SV. 


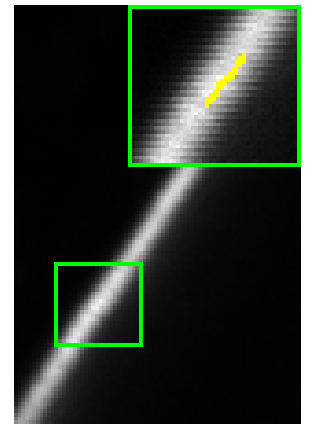

$\mathrm{PC}$

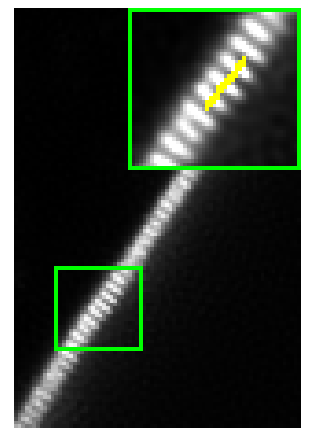

ISM-RL

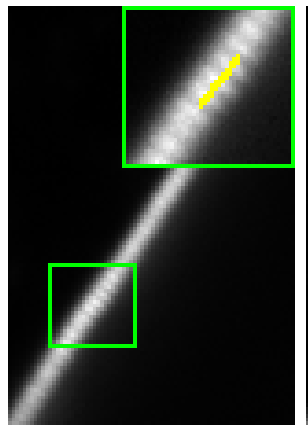

ISM

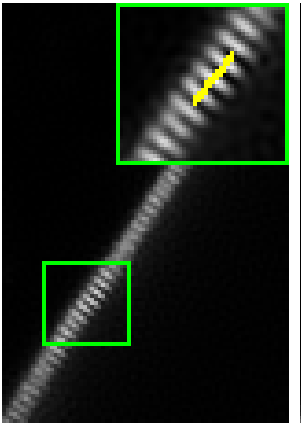

ISM-W

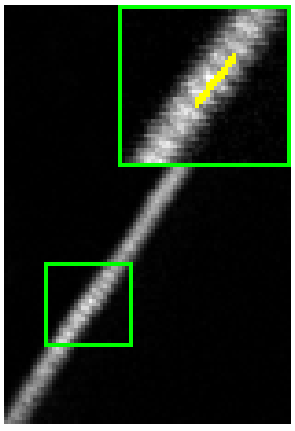

IFED

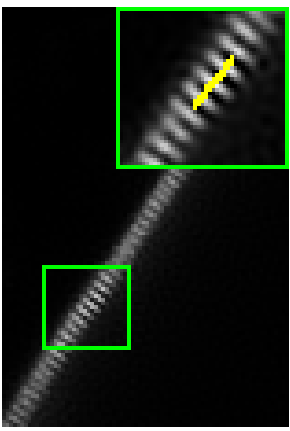

ISM-SV

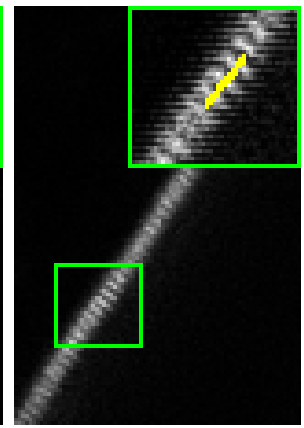

ISFED

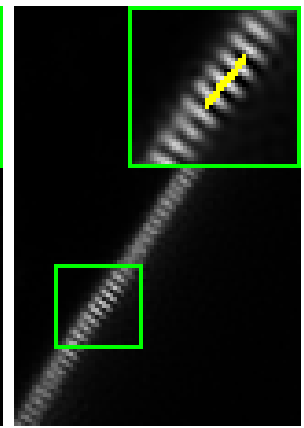

SV

Figure 7: Reconstruction results obtained on a $C$. elegans sample expressing the ERM-1::mNeonGreen fusion protein for all the tested methods. The reference profile line is displayed with a yellow line is plotted in Fig. 8 


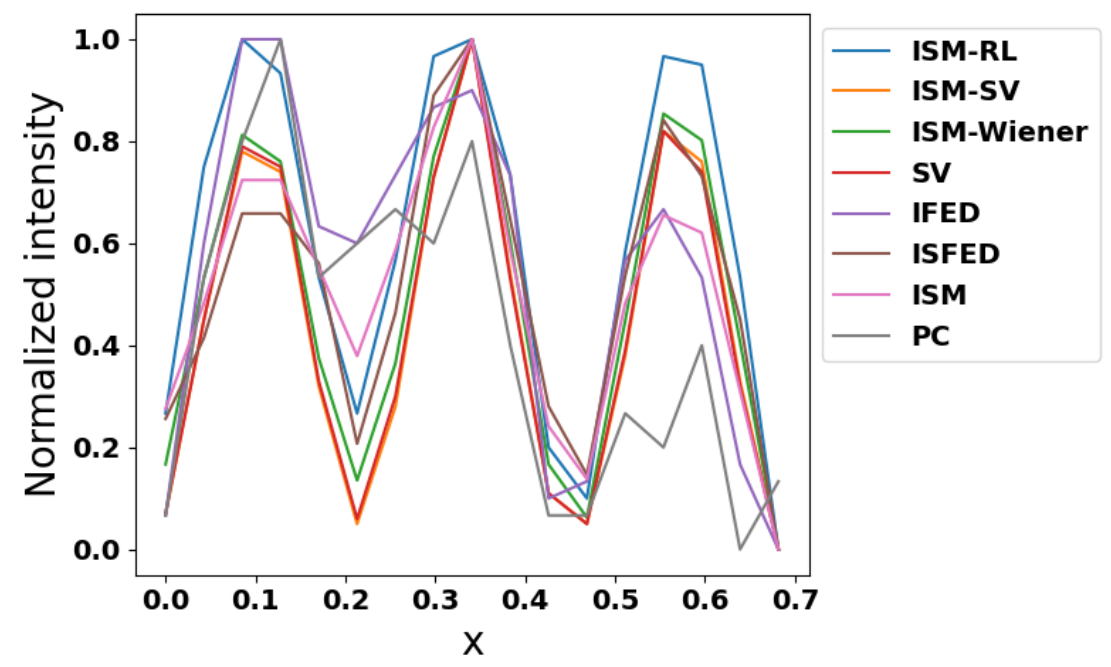

Figure 8: Profile along the green line of Fig. 7 for each reconstruction method. The $x$ axis is graduated in microns as the distance from the line starting point. The microvilli are not visible using the PC method. For the other methods the measured FWHM are: $131 \mathrm{~nm}$ for ISM, $131 \mathrm{~nm}$ for IFED, $141 \mathrm{~nm}$ for ISFED, 140 for ISM-RL, 127 for ISM-W, 115 for ISM-SV and 115 for SV.

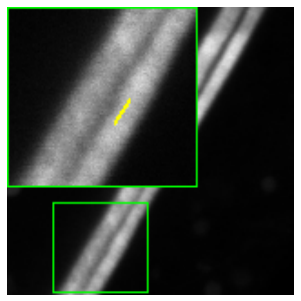

Speed \#1

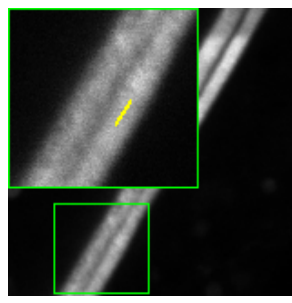

Speed \#2

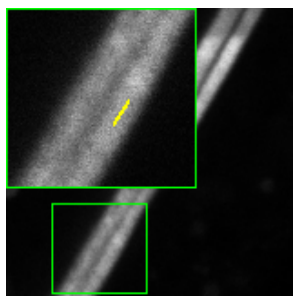

Speed \#3

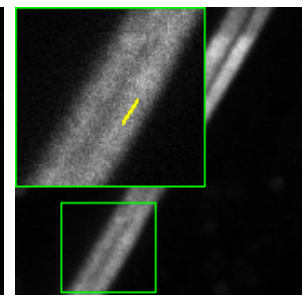

Speed \#4

Figure 9: Images of a $C$. elegans sample expressing the ERM-1::mNeonGreen fusion protein using using four different scanning speed from slow to fast. The pixel dwell time for speed $\# 1$ to $\# 4$ are $1.77 .10^{-4} s, 1.06 .10^{-4} s, 5.31 .10^{-5} s$ and $2.65 .10^{-5} s$. The yellow line show the profile used to measure the contrast of Fig 10 


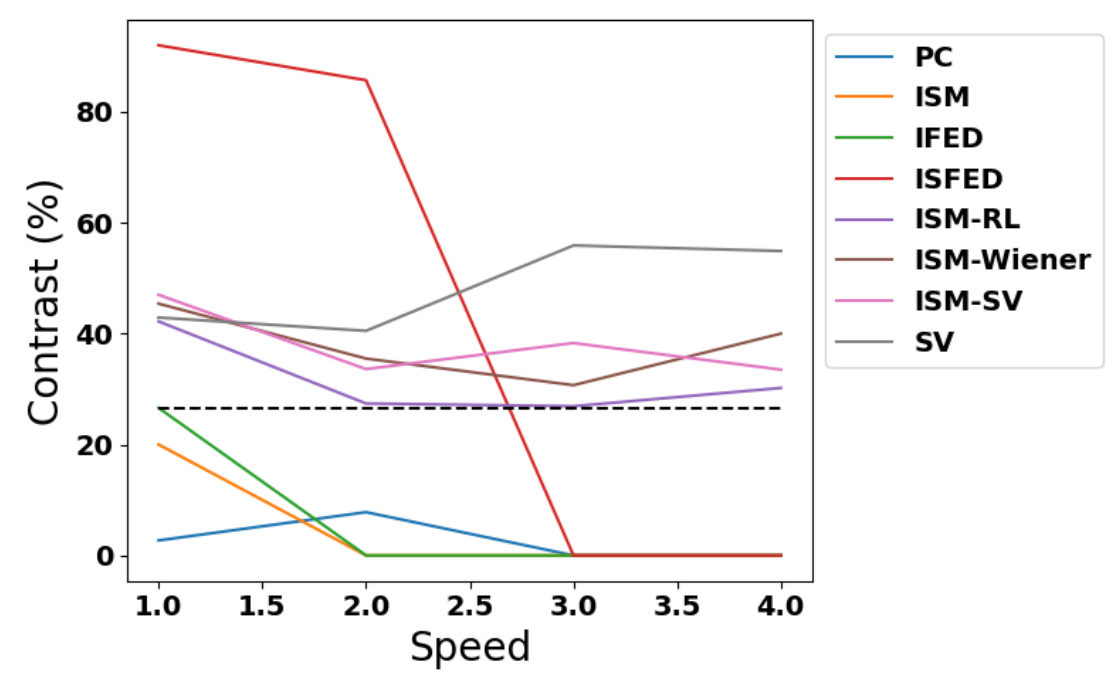

Figure 10: Plot of the measured intensity contrast in percentage between microvilli for each reconstruction method. The contrast is measured using a profile located at the yellow line plotted in Fig 9 . 